\title{
Variabilidade Interobservador no Diagnóstico Histológico dos Pólipos Colorretais
}

\author{
Interobserver Variability In The Histological Diagnosis \\ Of Colorectal Polyps
}

\author{
MARLISE MELLO CERATO ${ }^{1}$;NILO LUIZ CERATO ${ }^{2}$;LUISE MEURER ${ }^{3} ;$ MARIA ISABEL EDELWEISSA $^{4}$; \\ ANTÔNIO CARLOS PÜTTEN ${ }^{5}$;IANE GOLBSPAN ${ }^{6}$
}

\begin{abstract}
${ }^{1}$ Preceptora do Serviço de Coloproctologia do Hospital Ernesto Dornelles (HED), ${ }^{2}$ Chefe e Preceptor do Serviço de Coloproctologia do HED, ${ }^{3}$ Professora Doutora do Serviço de Patologia do HCPA, ${ }^{4}$ Professora Doutora do Serviço de Patologia do HCPA, ${ }^{5}$ Professor Doutor do Serviço de Patologia do HCPA, ${ }^{6}$ Patologista do Serviço de Patologia do HCPA e do HED - Porto Alegre, Rio Grande do Sul - Brasil.
\end{abstract}

CERATO MM, CERATO NL, MEURER L, EDELWEISS MI, PÜTTEN AC, GOLBSPAN L. Variabilidade Interobservador no Diagnóstico Histológico dos Pólipos Colorretais. Rev bras Coloproct, 2007;27(1): 007-015.

RESUMO: O manejo clínico dos pacientes com pólipos colorretais é principalmente baseado na histologia das lesões removidas. Em conseqüência, o diagnóstico histológico tem um papel muito importante na decisão terapêutica e a uniformidade de interpretação dos diferentes laudos de patologia é essencial. Apesar destas relevantes implicações, poucos estudos existem avaliando a variabilidade interobservador na elucidação dessa doença e a concordância não é considerada satisfatória. Objetivo: avaliar a variabilidade interobservador no diagnóstico histológico dos pólipos colorretais. Metodologia: foram avaliados 230 pólipos colorretais no Serviço de Patologia do Hospital de Clínicas de Porto Alegre (HCPA). Quatro patologistas examinaram todas as lâminas de forma independente e "cega", ou seja, sem conhecimento do diagnóstico elaborado pelo seu colega. As lesões colorretais foram classificadas em relação ao diagnóstico: pólipo e carcinoma invasivo e quanto ao tipo de pólipo: adenomatoso versus hiperplásico. Nos adenomas foram avaliados o tipo histológico (tubular, túbulo-viloso e viloso) e o grau de displasia (baixo e alto grau). Resultados: o Kappa médio, em relação ao tipo de lesão, foi de 0,794, considerado moderado. Quanto ao tipo de pólipo, o Kappa médio foi 0,852, ou seja, uma ótima concordância. Em relação aos adenomas, no que se refere ao tipo histológico, obteve-se um Kappa médio, fraco de 0,291, e na avaliação do grau de displasia o Kappa médio foi regular com valor de 0,420. Conclusão: o índice de concordância, entre os quatro observadores foi considerado de moderado a ótimo no tipo de lesão e de pólipo, porém a variabilidade foi grande na avaliação dos adenomas, tanto no que concerne ao tipo histológico quanto ao grau de displasia com um Kappa de fraco a regular.

Descritores: cólon; pólipos; adenomas; displasia; variabilidade; Kappa.

*Agradecimento ao FIPE (Fundo de Incentivo à Pesquisa e Eventos) pela tradução.

Trabalho realizado no Hospital de Clínicas de Porto Alegre (HCPA). Serviço de Patologia - Porto Alegre - RS - Brasil.

$\overline{\text { Recebido em 02/10/2006 }}$

Aceito para publicação em 08/02/07 
Rev bras Coloproct

Janeiro/Março, 2007
Variabilidade Interobservador no Diagnóstico

Histológico dos Pólipos Colorretais

Marlise Mello Cerato e Cols.
Vol. 27

$\mathbf{N}^{\mathbf{0}} 1$

\section{INTRODUÇÃO}

Os pólipos colorretais são freqüentes na população em geral. Contudo, existem dois tipos epiteliais mais comuns, os hiperplásicos e os adenomatosos ${ }^{1,2}$. Estes pólipos epiteliais são o resultado da perda do equilíbrio entre a proliferação celular nas criptas e a esfoliação, seja por excesso da primeira ou diminuição da segunda ${ }^{3}$. Os pólipos hiperplásicos, apesar de apresentarem algumas mutações genéticas, são considerados, não-neoplásicos ${ }^{4}$. O pólipo adenomatoso consiste na neoplasia intraepitelial ${ }^{4}$. Morfologicamente, os adenomas podem ser classificados quanto à arquitetura celular e o grau de atipia ${ }^{3}$. Em relação à arquitetura, segundo a percentagem do componente viloso, são classificados em tubular (menos de 20\%), túbulo-viloso (20-80\%) e viloso (mais de $80 \%$ ). Os adenomas apresentam anormalidades na arquitetura, citologia e diferenciação que, agrupadas, levam o nome de displasia. Todas essas lesões, por definição, são displásicas e apresentam alterações citológicas e arquiteturais que vão se sobrepondo até culminar no câncer colorretal ${ }^{5,6}$.

Os pólipos com elementos displásicos confinados ao epitélio ou à lâmina própria da mucosa são definidos como benignos. Por outro lado, quando existe invasão da submucosa o termo carcinoma invasivo é apropriado ${ }^{5,7-13}$. Os adenomas podem apresentar alterações displásicas de leves a severas dependendo do grau de complexidade vilosa ou glandular, extensão da estratificação nuclear e severidade das anormalidades encontradas ${ }^{4}$. No consenso de Viena (2002), os termos adenoma e displasia foram substituídos por neoplasia intra-epitelial (NIE), a qual é categorizada em dois graus, baixo e alto ${ }^{14,15}$. Na prática clínica o carcinoma "in situ" (CIS) e o carcinoma intramucoso, segundo a própria OMS (Organização Mundial da Saúde) ${ }^{4}$, devem ser classificados como displasia ou neoplasia intra-epitelial de alto grau, pois as repercussões dessas lesões são iguais, ou seja, nenhuma delas tem o potencial de enviar metástases para outros órgãos e/ou linfonodos $5,8-13,16$.

O diagnóstico anatomopatológico é que vai ditar o manejo clínico dos pacientes com lesões colorretais, sejam pólipos ou carcinomas invasivos. Assim, a precisão diagnóstica, isto é, mesmos achados levando a um mesmo diagnóstico, é fundamental para que se possa oferecer a melhor opção terapêutica ${ }^{17}$. O objetivo deste estudo foi avaliar a variabilidade interobservador entre quatro patologistas do Serviço de Patologia do HCPA no diagnóstico histológico dos pólipos colorretais.

\section{METODOLOGIA}

Foram analisados 230 pólipos colorretais de pacientes submetidos à endoscopia digestiva baixa no HCPA, no período de um ano: de julho de 2004 a julho de 2005. Os casos foram consecutivos, eliminando-se os que se enquadravam nos critérios de exclusão: os pólipos inflamatórios ou hamartomatosos, as poliposes intestinais, as doenças inflamatórias intestinais, o carcinoma colorretal não-polipóide e as biópsias colorretais com outros fins que não a excisão de pólipo. As lesões foram avaliadas de forma independente e "cega" por quatro patologistas do HCPA com experiência em patologia do trato gastrointestinal, os quais, rotineiramente, fazem diagnósticos anatomopatológicos, tanto neste serviço quanto em laboratórios privados. Todas as lâminas foram coradas com hematoxilina-eosina. Não houve treinamento ou padronização dos critérios classificatórios pelos patologistas.

As lesões foram, num primeiro momento, divididas em pólipos e carcinomas invasivos. Os pólipos foram divididos em hiperplásicos e adenomatosos. De acordo com a percentagem do componente viloso, os adenomas foram subdivididos em tubular (menos de $20 \%$ ), túbulo-viloso (20-80\%) e viloso (mais de $80 \%$ )

${ }^{4}$. Utilizou-se o sistema que divide a displasia em baixo e alto grau ${ }^{4,18,19}$. As características mais importantes na avaliação do grau de displasia foram a arquitetura tecidual, as alterações nucleares e a diferenciação citoplasmática, as quais vão desde mínimas até grosseiras, definindo, assim, as categorias ${ }^{20}$.

Os carcinomas invasivos foram classificados segundo o grau de diferenciação em bem diferenciado, moderadamente diferenciado, pouco diferenciado ou indiferenciado ${ }^{4,21}$. Nessas lesões, também foram analisadas a existência ou não de infiltração da base do pólipo, invasão vascular e linfática.

\section{Análise estatística}

Trata-se de um estudo transversal, prospectivo e "cego" entre os patologistas. A pesquisa foi quantitativa com enfoque descritivo. $\mathrm{O}$ tamanho da amostra ficou estabelecido em 230 pólipos, considerando um nível de significância de 5\%, um poder de teste de $80 \%$ e um erro máximo admissível de $10 \%$. A análise dos dados foi gerada no programa SPSS, versão 11.5.

Para avaliar a variabilidade interobservador utilizou-se o índice Kappa de Cohen. A concordância 
segue a orientação da literatura: Kappa $<0,10$ ausente; de 0,11 a 0,40 fraca; de 0,41 a 0,60 discreta (regular); de 0,61 a 0,80 moderada; de 0,81 a 0,99 substancial (ótima) e 1,00 concordância perfeita ${ }^{22-28}$.

\section{RESULTADOS}

\section{invasivo)}

Tipo de diagnóstico (pólipo e carcinoma

Sobre a comparação dos percentuais apresentados pelos quatro patologistas, não foi identificada diferença estatística significativa para o tipo de diagnóstico (Tabela 1). Na concordância dos diagnósticos interobservadores o índice Kappa oscilou de 0,000 a 0,798, ou seja, de ausente a moderado (Tabela 2 e Figura 1). O Kappa médio foi de 0,794 (IC95\%=0,314-1,274), istoé, os examinadores concordaram em 181 dos 230 casos das lesões colorretais avaliadas na amostra.

\section{adenomatoso) \\ Tipo histológico de pólipo (hiperplásico e} A distribuição dos percentuais apresentada pelos examinadores para o tipo histológico de pólipo, não apresentou diferença significativa (Tabela 1). Na concordância interobservador foi obtida a classificação ótima em todas as comparações com um Kappa de 0,821 a 0,954 (Tabela 2 e Figura 1). O Kappa médio foi de 0,852 (IC95\% = 0,787-0,916), ou seja, os pato- logistas encontraram o mesmo diagnóstico em 186 dos 228 casos, em média, avaliados na amostra.

Tipo histológico de adenoma (tubular, túbulo-viloso e viloso)

Nos percentuais atribuídos pelos patologistas foi identificada alta variabilidade, implicando em diferença estatística significativa, principalmente em relação à porcentagem apresentada pelo observador um (Tabela 1). $\mathrm{O}$ índice de concordância interobservador assumiu valores de 0,254 a 0,493 , ou seja, concordância de fraca a regular (Tabela 2 e Figura 1). O Kappa médio estimado foi de 0,291 (IC95\% = 0,166 - 0,416), isto é, os patologistas coincidiram no diagnóstico de 35 dos 169 casos, em média, avaliados na amostra.

\section{grau)}

\section{Grau de displasia do adenoma (baixo e alto}

Dentre os percentuais que cada patologista apresentou, foi identificada diferença estatística significativa do observador dois em relação aos demais (Tabela 1). O índice de concordância interobservador oscilou de 0,068 a 0,566, ficando a classificação de ausente a regular (Tabela 2 e Figura 1). O Kappa médio estimado para o grau de displasia entre os quatro observadores foi de 0,420 (IC95\% = 0,196-0,644), o que equivale a diagnósticos coincidentes em 63 dos 169 casos, em média, apresentados na amostra.

Tabela 1 - Caracterização da amostra de acordo com os quatro patologistas.

\begin{tabular}{|c|c|c|c|c|c|c|c|c|}
\hline \multirow[t]{2}{*}{ Diagnósticos } & \multicolumn{2}{|c|}{ Obs. 1} & \multicolumn{2}{|c|}{ Obs. 2} & \multicolumn{2}{|c|}{ Obs. 3} & \multicolumn{2}{|c|}{ Obs. 4} \\
\hline & $\bar{n}$ & $\%$ & $\mathbf{n}$ & $\%$ & $\overline{\mathbf{n}}$ & $\%$ & $\mathbf{n}$ & $\%$ \\
\hline \multicolumn{9}{|c|}{ Tipo de lesão } \\
\hline Pólipo & 229 & 99,6 & 228 & 99,1 & 227 & 98,7 & 230 & 100,0 \\
\hline Carcinoma invasivo & 1 & 0,4 & 2 & 0,9 & 3 & 1,3 & 0 & 0,0 \\
\hline \multicolumn{9}{|c|}{ Tipo histológico } \\
\hline Hiperplásico & 58 & 25,3 & 61 & 26,8 & 61 & 26,9 & 58 & 25,2 \\
\hline Adenomatoso & 171 & 74,7 & 167 & 73,2 & 166 & 73,1 & 172 & 74,8 \\
\hline \multicolumn{9}{|c|}{ Tipo histológico pólipo adenomatoso } \\
\hline Tubular & 149 & 87,1 & 121 & 72,5 & 90 & 54,2 & 110 & 63,9 \\
\hline Túbulo-viloso & 15 & 8,8 & 45 & 26,9 & 65 & 39,2 & 55 & 32,0 \\
\hline Viloso & 7 & 4,1 & 1 & 0,6 & 11 & 6,6 & 7 & 4,1 \\
\hline \multicolumn{9}{|c|}{ Grau de displasia pólipo adenomatoso } \\
\hline Baixo grau & 158 & 92,4 & 146 & 87,4 & 151 & 91,0 & 165 & 95,9 \\
\hline Alto grau & 13 & 7,6 & 21 & 12,6 & 15 & 9,0 & 7 & 4,1 \\
\hline
\end{tabular}


Tabela 2: Estimativa do índice Kappa $(k)$ na comparação dos observadores dois a dois nas classificações da amostral.

\begin{tabular}{|c|c|c|c|c|}
\hline \multirow{3}{*}{$\begin{array}{l}\text { Patologistas } \\
\text { Obs. } 1 \text { / Obs. } 2\end{array}$} & \multicolumn{4}{|c|}{ Kappa (IC95\%) } \\
\hline & \multicolumn{2}{|c|}{ Tipo de diagnóstico } & \multicolumn{2}{|c|}{ Tipo histológico de pólipo } \\
\hline & $0,665^{\mathrm{B}}$ & $(0,046-1,000)$ & $0,943^{\mathrm{A}}$ & $(0,895-0,991)$ \\
\hline Obs. 1 / Obs. 3 & $0,497^{\mathrm{B}}$ & $(0,000-1,000)$ & $0,852^{\mathrm{A}}$ & $(0,773-0,930)$ \\
\hline Obs. 1 / Obs. 4 & $0,000^{*}$ & $(0,000-0,258)$ & $0,954^{\mathrm{A}}$ & $(0,910-0,997)$ \\
\hline Obs. 2 / Obs. 3 & $0,798^{\mathrm{A}}$ & $(0,411-1,000)$ & $0,821^{\mathrm{A}}$ & $(0,735-0,906)$ \\
\hline Obs. 2 / Obs. 4 & $0,000 *$ & $(0,000-0,258)$ & $0,943^{\mathrm{A}}$ & $(0,895-0,991)$ \\
\hline Obs. 3 / Obs. 4 & $0,000 *$ & $(0,000-0,258)$ & $0,829^{\mathrm{A}}$ & $(0,746-0,912)$ \\
\hline Patologistas & \multicolumn{2}{|c|}{ Tipo pólipo adenomatoso } & \multicolumn{2}{|c|}{ Grau de displasia } \\
\hline Obs. 1 / Obs. 2 & $0,446^{\mathrm{B}}$ & $(0,311-0,581)$ & $0,566^{\mathrm{A}}$ & $(0,355-0,777)$ \\
\hline Obs. 1 / Obs. 3 & $0,254^{\mathrm{B}}$ & $(0,152-0,355)$ & $0,332^{\mathrm{A}}$ & $(0,080-0,583)$ \\
\hline Obs. 1 / Obs. 4 & $0,422^{\mathrm{B}}$ & $(0,292-0,551)$ & $0,502^{\mathrm{A}}$ & $(0,224-0,779)$ \\
\hline Obs. 2 / Obs. 3 & $0,365^{\mathrm{B}}$ & $(0,235-0,494)$ & $0,437^{\mathrm{A}}$ & $(0,219-0,656)$ \\
\hline Obs. 2 / Obs. 4 & $0,493^{\mathrm{B}}$ & $(0,362-0,624)$ & $0,191^{\mathrm{B}}$ & $(0,122-0,648)$ \\
\hline Obs. 3 / Obs. 4 & $0,398^{\mathrm{B}}$ & $(0,269-0,527)$ & $0,068^{\mathrm{C}}$ & $(0,000-0,256)$ \\
\hline
\end{tabular}

A: Significância aproximada $p<0,0001$

B: Significância aproximada $p<0,001$

$C: p>0,05$

$1 * k<0:$ concordância, além de nula, é menor que aleatoriedade, ié, discordância $;>1:$ Concordância se deve a fatores aleatórios; $k=$ 0: concordância é igual à aleatoriedade.

\section{Carcinoma invasivo}

Em relação ao carcinoma invasivo, somente os três primeiros observadores encontraram este diagnóstico (Tabela 3).

Numa avaliação global dos diagnósticos que os quatro patologistas conferiram a cada um dos pólipos avaliados, chama a atenção que a categoria carcinoma invasivo teve concordância nula, já que um dos patologistas não diagnosticou nenhum caso desta etiologia. Já os outros três patologistas concordaram com este diagnóstico em apenas um dentre os três casos possíveis, resultando em uma acedência de 33,3\%.

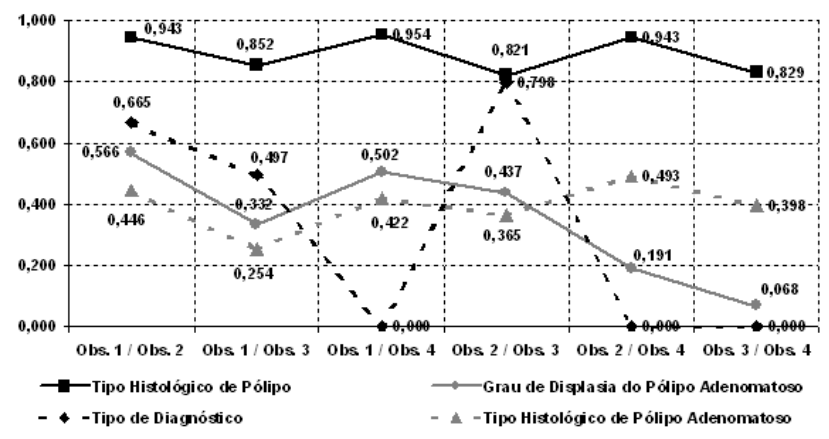

Figura 1 - Índice de concordância Kappa para os diagnósticos estudados nas comparações entre os quatro observadores.

\section{DISCUSSÃO}

O paradigma definidor do prognóstico de pacientes com lesões colorretais é o diagnóstico anatomopatológico com o qual vai se tomar a decisão terapêutica. Assim, é prioritário uniformizar a interpretação dos diferentes laudos da patologia ${ }^{1}$, como também conhecer a variabilidade de diagnósticos para uma mesma lesão.

No tipo de diagnóstico, as lesões são classificadas em pólipos e carcinomas invasivos. Os pólipos foram a grande maioria: $98,7-100 \%$ dos casos do presente estudo, e os carcinomas invasivos foram diagnosticados em 0,0 -1,3\% dos casos. Em um estudo da Cleveland Clinic, onde foram avaliados 1523 pólipos adenomatosos, foram encontrados 41 casos de carcinomas invasivos $(2,7 \%)$, o que se assemelha aos resultados desta pesquisa ${ }^{21}$. Outros autores relataram a presença de carcinoma invasivo em $0,2-8,3 \%$ dos pólipos ${ }^{29-31}$.

Neste estudo, obteve-se uma grande variabilidade quanto ao tipo de diagnóstico, se for mencionado o Kappa nas comparações interobservadores, pois este variou de 0,000 a 0,798 , ou seja, de ausente a modera- 
Tabela 3: Comparação das classificações do carcinoma invasivo por observador ${ }^{2}$.

\begin{tabular}{lcccccc}
\hline Características & \multicolumn{2}{c}{ Obs. 1 } & \multicolumn{2}{c}{ Obs. 2 } & \multicolumn{2}{c}{ Obs. 3 } \\
\cline { 2 - 6 } & Divisão & Freq. & Divisão & Freq. & Divisão & Freq. \\
\hline Grau de Diferenciação & Moderado & 1 & Bem & 2 & Bem & 2 \\
Infiltração da base pólipo & --- & --- & --- & --- & Moderado & 1 \\
& Sim & --- & Sim & --- & Sim & 2 \\
Invasão vascular & Não & 1 & Não & 2 & Não & 1 \\
& Sim & 1 & Sim & --- & Sim & 1 \\
Invasão linfática & Não & --- & Não & 2 & Não & 2 \\
& Sim & 1 & Sim & -- & Sim & 2 \\
& Não & --- & Não & 2 & Não & 1 \\
\hline
\end{tabular}

$2 *$ O observador quatro não diagnosticou carcinoma invasivo.

Orientação: retrato

Ordem no texto: $1^{a}$

Nome do autor: Marlise Mello Cerato

Título do artigo: Variabilidade Interobservador no Diagnóstico Histológico dos Pólipos Colorretais (The Interobserver Variability in the Histological Diagnosis of the Colorectal Polyps) - Trabalho realizado no Hospital de Clínicas de Porto Alegre (HCPA) Serviço de Patologia.

do. Por outro lado, com base no percentual de concordância relativa, pode-se dizer que os observadores concordaram entre si quase que plenamente quanto ao tipo de diagnóstico dos pólipos, pois os valores da concordância relativa se apresentaram acima de $99,0 \%$. O índice de concordância médio entre os quatro observadores foi de 0,794 (IC95\% = 0,314-1,274): todos os examinadores deram um mesmo diagnóstico em 181 dos 230 casos avaliados.

Um estudo italiano, multicêntrico, que avaliou a variabilidade interobservador no diagnóstico histológico dos pólipos colorretais, evidenciou uma concordância (Kappa) de 0,78, isto é, moderada, comparando presença ou ausência de carcinoma invasivo ${ }^{1}$. Nesta pesquisa realizada pela autora e colegas, alguns observadores não obtiveram concordância e outros alcançaram um resultado semelhante ao da literatura: um Kappa moderado.

O tratamento de primeira escolha para os adenomas com displasia de alto grau ou carcinomas intramucosos é a polipectomia ou mucosectomia endoscópica ${ }^{32}$. A necessidade de tratamento cirúrgico complementar depende do risco de metástases linfonodais ou viscerais ${ }^{32}$. O surgimento destas metástases torna-se possível a partir da invasão da camada submucosa quando a lesão passa a ser conceituada como carcinoma invasivo ${ }^{4}$. A ocorrência de implantes metastáticos linfonodais nesse contexto, varia de 3,6-16,2\% e de metástases viscerais, de aproximadamente $3,0 \%{ }^{32-34}$. Para que não seja necessário o tratamento complementar no carcinoma invasor, ele deve ser totalmente excisado, ter avaliação completa da profundidade da invasão, ser bem ou moderadamente diferenciado, não ter invasão linfo-vascular nem comprometimento das margens de ressecção ${ }^{13}$.

Nesta pesquisa, em relação ao tipo histológico, os pólipos foram divididos em hiperplásicos e adenomatosos. O percentual de pólipos hiperplásicos variou de $25,2-26,9 \%$ com mediana de $26,0 \%$, o que vem ao encontro da literatura, que relata em estudos de rastreamento de colonoscopias, uma percentagem de 9,0-34,0\% deste tipo histológico de pólipo ${ }^{35-37}$. Por outro lado, os pólipos adenomatosos nesta amostra variaram de $73,1-74,8 \%$ e mediana de $73,9 \%$, enquanto que nos estudos de colonoscopias tiveram uma prevalência menor de 24,0-48,0\% ${ }^{35-37}$. Um trabalho multicêntrico italiano sobre avaliação interobservador no diagnóstico histológico dos pólipos colorretais, onde foram analisados 100 pólipos colorretais, $20 \%$ foram classificados como hiperplásicos e $80 \%$, como adenomatosos. Tais percentuais não apresentam diferença significativa quando comparados aos resultados da amostra desta pesquisa ${ }^{1}$.

A variabilidade interobservador dos quatro patologistas no diagnóstico histológico dos pólipos colorretais, medida pelo índice Kappa de Cohen, foi classificada como ótima, ou seja, houve poucas discordâncias em relação ao tipo de pólipo. O Kappa variou, na comparação dos patologistas dois a dois, de 0,821 a 0,954 e este resultado é concordante com ou- 
tros estudos ${ }^{38,39}$. Em relação aos quatro patologistas, o Kappa médio foi de 0,852 (IC95\% = 0,787-0,916), isto é, encontraram o mesmo diagnóstico em 186 casos de pólipos dos 228 casos analisados, em média, o que se assemelha ao estudo de Constantini et al. os quais encontraram um Kappa médio de 0,89 para esta categoria ${ }^{1}$.

Estes resultados são de extrema importância, pois os pólipos hiperplásicos, apesar de apresentarem algumas mutações, como no K-ras, são considerados não-neoplásicos ${ }^{4}$. Tais pólipos, quando pequenos (menores que 5-7 mm), com aspectos macroscópicos típicos, sem depressão na superfície e com padrão de criptas tipo II podem ser deixados "in situ", não havendo necessidade de biópsia ou tratamento endoscópico, o que diminui riscos e custos dos exames endoscópicos 40-45. Por outro lado, os adenomas são neoplasias intraepiteliais que apresentam anormalidades na arquitetura, citologia e diferenciação que, agrupadas, levam o nome de displasias ${ }^{46}$. São lesões pré-cancerosas que sofrem mutações genéticas dos genes APC, K-ras, DCC e p53, dentre outras, envolvidas na sequiência adenoma-carcinoma ${ }^{4-6}$.

Os pólipos adenomatosos foram classificados quanto ao tipo histológico (tubular, túbulo-viloso e viloso) e quanto ao grau de displasia (baixo e alto grau).

Em relação ao tipo histológico dos pólipos adenomatosos, encontrou-se 54,2-87,1\% de adenomas tubulares com mediana de $68,2 \% ; 8,8-39,2 \%$ de túbulovilosos com mediana de $29,4 \%$ e $0,6-6,6 \%$ de vilosos com mediana de $4,1 \%$. Estes achados apresentam valores próximos aos obtidos no National Polyp Study, realizado em Nova York em 1990, no que se refere à amplitude dos percentuais encontrados. Este estudo americano constituiu-se em um ensaio clínico randomizado que analisou 3371 pólipos adenomatosos de 1867 pacientes ${ }^{47}$. Foram avaliados fatores de riscos dos pacientes e dos pólipos e sua associação com a displasia de alto grau. O tamanho do adenoma e a extensão do componente viloso, assim como a idade acima de 60 anos são fatores de risco independentes que estão relacionados com a atipia de alto grau. Dos pólipos analisados neste estudo $87 \%$ eram adenomas tubulares, $8 \%$, túbulo-vilosos e 5\%, vilosos ${ }^{47}$. Apesar dos resultados semelhantes, a comparação deste trabalho com o que se realizou, mostra diferença estatisticamente significativa $(\mathrm{p}<0,05)$, ou seja, os valores detectados pelo National Polyp Study foram superiores às medianas encontradas nesta pesquisa.
No que se refere ao tipo histológico dos adenomas, a variabilidade foi grande: obteve-se, na comparação dos patologistas dois a dois, um Kappa que variou de 0,254 a 0,493, caracterizando uma concordância de fraca a regular. Na concordância dos quatro patologistas o Kappa médio foi de 0,291 (IC95\% $=0,166-0,416$ ), onde em apenas 35 dos 169 casos analisados, em média, foi dado o mesmo diagnóstico por todos os observadores. Terry et al. avaliaram 190 pólipos adenomatosos e relataram um Kappa de 0,48 para o tipo histológico de adenoma, dividindo em tubular, túbuloviloso e viloso ${ }^{19}$. Estes resultados evidenciam uma concordância nesta categoria superior a que foi relatada neste estudo. Em outro trabalho, de Yoon $\mathrm{H}$ et al., foi encontrado um Kappa de 0,46 no tipo histológico de adenoma com uma variabilidade menor que a desta pesquisa ${ }^{48}$. Nessa categoria, os resultados deste trabalho mostraram uma grande variabilidade, mas a repercussão não é relevante de uma forma direta, pois a conduta não é modificada somente pela diferença do tipo histológico. Porém, sabe-se que quanto maior o tamanho do pólipo e quanto maior o componente viloso, mais chance terá de apresentar displasia de alto grau e carcinoma invasivo ${ }^{3}$.

Em relação ao grau de displasia, utilizou-se a classificação que divide em duas categorias, baixo e alto grau. Foram diagnosticados $87,4-95,9 \%$ adenomas com displasia de baixo grau com mediana de $91,7 \%$ e $4,1-12,6 \%$ adenomas com displasia de alto grau com mediana de $8,3 \%$. Segundo alguns autores, a displasia de alto grau está presente em $2-5 \%$ dos adenomas ${ }^{3,20}$.

No que concerne ao grau de displasia, encontrou-se um Kappa de ausente a regular que variou, nas comparações dos observadores dois a dois, de 0,068 a 0,566 , evidenciando uma concordância baixa entre os patologistas. O Kappa médio foi de 0,420 (IC95\% = 0,196-0,644), isto é, os quatro examinadores concordaram em 63 dos 169 casos, em média, avaliados nesta categoria. Os resultados da pesquisa apresentaram uma concordância superior à descrita por Yoon $\mathrm{H}$ et al. $(0,34)$ nesta categoria ${ }^{48}$. Por outro lado, obteve-se uma variabilidade maior que a relatada por Terry et al., os quais descrevem um Kappa de 0,69 em relação ao grau de displasia dividida em duas categorias, baixo e alto grau ${ }^{19}$.

Fenger et al. publicaram um trabalho avaliando a reprodutibilidade intra e interobservador na avaliação do grau de displasia dos adenomas colorretais, comparando dois sistemas classificatórios: Konishi- 
Morson System (KMS) e Kozuka ${ }^{49}$. O KMS divide a displasia em três graus: leve, moderada e severa, podendo ser utilizadas duas categorias, leve/moderada versus severa. O KMS utiliza para definir a graduação: configuração tubular, polaridade nuclear, orientação, estrutura e conteúdo de mucina, dentre outros critérios. O sistema Kozuka descreve cinco categorias, sendo consideradas displásicas da III-V, o critério que define o grau é a pseudoestratificação epitelial ${ }^{49}$. Segundo Fenger et al., o Kozuka grau $\mathrm{V}$ foi subdividido em três graus e denominado Extended Kozuka System (EKS), incluindo o carcinoma in situ (CIS) e o carcinoma intramucoso (IMC). O EKS pode utilizar dois graus, um para leve/ moderada/severa e outro para CIS/IMC. Estes autores publicaram resultados surpreendentes quanto ao grau de displasia, comparando estes dois sistemas classificatórios quando modificados para duas categorias, conforme foi descrito anteriormente, tiveram um Kappa de 0,76 para o EKS e 0,80 para o KMS ${ }^{49}$.

Uma grande variabilidade em relação ao grau de displasia não ocorre somente com a neoplasia colorretal, mas também há relatos de Kappa com variações de 0,20 a 0,45 em estudos de mama, colo uterino, próstata e lesões orais não-invasivas ${ }^{19}$. Essa divisão em displasia de baixo e alto grau é importante, pois a presença da displasia de alto grau tem uma forte correlação com o carcinoma invasivo. A percentagem de adenomas com alterações displásicas severas e com carcinoma invasivo aumenta significativamente com o tamanho do pólipo, a arquitetura vilosa, a multiplicidade de adenomas e com a idade maior de 60 anos ${ }^{3}$.

Diferentes estratégias são propostas para diminuir a variabilidade interobservador no diagnóstico histológico dos pólipos colorretais. Dentre elas, o estabelecimento de protocolos que devem ser seguidos como guias para se chegar a um diagnóstico, a revisão das lâminas por dois ou mais patologistas nos casos de displasia de alto grau ou carcinoma invasivo, reuniões anatomoclínicas, discussões de casos com microscópio de várias cabeças ou sistema de vídeo-microscópio para que as consequiências possam ser minimizadas e o paciente tenha um correto diagnóstico e o melhor tratamento ${ }^{1,50-53}$.

\section{CONCLUSÃO}

Entre os quatro patologistas, obteve-se um índice de concordância com classificação de moderado a ótimo em relação ao tipo de lesão e de pólipo colorretal. Quanto ao tipo histológico dos adenomas e grau de displasia, a variabilidade foi acentuada com um Kappa de fraco a regular.

De uma maneira geral, levando-se em consideração as coincidências identificadas em todos os resultados referentes às quatro variáveis investigadas no estudo, verificou-se que a concordância geral observada foi alcançada em 117 dos 230 casos avaliados pelos patologistas na amostra, ou seja, eles concordaram plenamente em 50,8\% dos diagnósticos.

Com isso, podemos concluir, que existe uma necessidade veemente de padronização dos critérios classificatórios, treinamento dos profissionais e maior troca de informações visando uma melhor interação de toda a equipe para melhorar a variabilidade interobservador dos patologistas em relação aos pólipos colorretais.

\footnotetext{
ABSTRACT: The clinical management of patients with colorectal polyps is mainly based on the histology of the removed lesions. Therefore, the histological diagnosis has a very important role in deciding the treatment and the uniform interpretation of the different pathology reports is essential. In spite of these relevant implications, there are only very few studies assessing the interobserver variability in such diagnosis and the concordance of reports among different examiners is not considered satisfactory. Objective: to assess interobserver variability in the pathology reports in the diagnosis of colorectal polyps. Method: at the Department of Pathology of HCPA [Hospital de Clínicas de Porto Alegre] 230 slides of colorectal polyps were examined by four independent pathologists "blindly", that is, the diagnosis given by their colleagues was not known. Colorectal lesions were classified according to the diagnosis as polyp or invasive cancer and to the polyp type (adenomatous or hyperplasic). The histological type of the adenomas (tubular, tubulovillous and villous) and the grade of dysplasia (high or low) were also assessed. Results: mean Kappa of the type of lesion was 0.794 , which is considered moderate. The mean Kappa of 0.852 for the type of polyp is considered excellent concordance. Regarding the histology of adenomas, the mean Kappa was 0.291, considered weak. The assessment of the degree of dysplasia showed a regular Kappa of $\mathbf{0 . 4 2 0}$. Conclusion: the concordance rate among the four pathologists was considered to be moderate to excellent for the type of lesion and of polyp but there was great variability in the assessment of adenomas both for the histological type and for dysplasia, showing a weak to regular Kappa.
}

Key words: colon, polyps, adenomas, dysplasia, variability, Kappa. 


\section{REFERÊNCIAS}

1. Costantini M, Sciallero S, Giannini A, Gatteschi B, Rinaldi P, Lanzanova $\mathrm{G}$ et al. Interobserver agreement in the histologic diagnosis of colorectal polyps: the experience of the multicenter adenoma colorectal study (SMAC). Journal of Clinical Epidemiology 2003; 56:209-14.

2. Lane N, Kaplan H, Pascal RR. Minute adenomatous and hyperplastic polyps of the colon: Divergent patterns of epithelial growth with specific associated mesenchymal changes. Contrasting roles in the pathogenesis of carcinoma. Gastroenterology 1971; 60:537-51.

3. Fenoglio - Preiser CM, Noffsinger AE, Stemmermann GN, Lantz PE, Listrom MB, Rilke FO. Carcinomas and Other Epithelial and Neuroendocrine Tumors of the Large Intestine. In: Fenoglio - Preiser CM, Noffsinger AE, Stemmermann GN, Lantz PE, Listrom MB, Rilke FO. Gastrointestinal Pathology An Atlas and Text. 2nd ed. Philadelphia: Lippincott Williams and Wilkins; 1999. 909-1068.

4. Hamilton SR, Vogelstein B, Kudo S, Riboli E, Nakamura S, Hainaut P. Carcinoma of the colon and rectum. In: Hamilton SR, Aaltonen LA. World Health Organization Classification of Tumours - Pathology and Genetics - Tumours of the Digestive System. 3rd ed. Lyon: IARCPress; 2000. 105-43.

5. Pascal RR. Dysplasia and Early Carcinoma in Inflammatory Bowel Disease and Colorectal Adenomas. Human Pathology 1994; 25(11):1160-71.

6. Muto T, Bussey HJ, Morson BC. The evolution of cancer of the colon and rectum. Cancer. 1975; 36: 2251-70.

7. Rex DK, Ulbright TM, Cummings OW. Coming to terms with pathologists over colon polyps with cancer or high-grade dysplasia. J Clin Gastroenterol 2005; 39(1):1-3.

8. Richards WO, Webb WA, Morris SJ, Davis RC, McDaniel L, Jones L et al. Patient management after endoscopic removal of the cancerous colon adenoma. Ann Surg 1987; 205(6):665-70.

9. Speroni AH, Meiss RP, Calzona C, Castelletto RH, Jmelnitzky A, Chopita N et al. Early Colorectal cancer- Follow-up after Endoscopic Polypectomy. Endoscopy 1988; 20:18-20.

10. Morson BC, Whiteway JE, Jones EA, Macrae FA, Williams CB. Histopathology and prognosis of malignant colorectal polyps treated by endoscopic polypectomy. Gut 1984; 25:437-44

11. Lane N, Kaye GI. Pedunculated adenomatous polyp of the colon with carcinoma, lymph node metastasis, and suture-line recurrence. Am J Clin Pathol 1967; 48:170-82.

12. Fenoglio-Preiser CM, Kay GI, Lane N. Distribution of human colonic lymphatics in normal, hyperplastic and adenomatous tissue, its relationship to metastasis from small carcinomas in pedunculated adenomas with two case reports. Gastroenterology 1973; 60:51-66.

13. Cooper HS, Deppisch LM, Kahn EI, Lev R, Manley PN, Pascal RR, et al. Pathology of the malignant colorectal polyp. Human Pathology 1998; 29:15-26.
14. Schlemper RJ, Riddell RH, Kato Y, Borchard F, Cooper HS, Dawsey SM. The Vienna Classification of gastrointestinal epithelial neoplasia. Gut 2000; 47(2): 251-5.

15. Dixon MF. Gastrointestinal epithelial neoplasia: Vienna revisited. Gut 2002; 51:130-1.

16. Rex DK, Alikhan M, Cummings O, Ulbright TM. Accuracy of pathologic interpretation of colorectal polyps by general pathologists in community practice. Gastrointestinal Endoscopy 1999; 50(4):468-74.

17. Foucar E. Error Identification: a surgical pathology dilemma. Am J Surg Pathol 1998; 22(1):1-5.

18. West B, Mitsuhashi T. Cancer or high-grade dysplasia? The present status of the application of the terms in colonic polyps. J Clin Gastroenterol 2005; 39(1):4-6.

19. Terry MB, Neugut AI, Bostick RM, Potter JD, Haile RW, Fenoglio-Preiser CM. Reliability in the classification of advanced colorectal adenomas. Cancer Epidemiology, Biomarkers and Prevention 2002; 11:660-3.

20. Morson BC, Dawson IMP, Day DW, Jass JR, Price AB, Shepherd NA et al. Epithelial tumours of the large intestine. In: Morson BC, Dawson IMP, Day DW, Jass JR, Price AB, Shepherd NA et al. Morson and Dawson's Gastrointestinal Pathology. 4th ed.Massachusetts: Blackwell Publishing; 2003. 551-609.

21. Cranley JP, Petras RE, Carey WD, Paradis K, Sivak MV. When is endoscopic polypectomy adequate therapy for colonic polyps containing invasive carcinoma? Gastroenterology 1986; 91(2):419-27.

22. Landis JR, Koch GG. The measurement of observer agreement for categorical data. Biometrics 1977; 33:159-74.

23. Silva EF, Pereira MG. Avaliação das estruturas de concordância e discordância nos estudos de confiabilidade. Rev Saúde Pública 1998; 32:383-93.

24. Brennan P, Silman A. Statistical methods for assessing observer variability in clinical measures. BMJ 1992; 304:1491-4.

25. Cross SS. Kappa statistics as indicators of quality assurance in histopathology and cytopathology. J Clin Pathol 1996; 49(7):597-9.

26. Cross SS. Grading and scoring in histopathology. Histopathology 1998; 33(2):99-106.

27. Fleiss JL. Statistical methods for rates and proportions.2nd ed. Nova York: Wiley; 2000.

28. Silcocks P. Some issues in observer error studies in pathology. J Pathol 1992; 168:255-6.

29. Wolff WI, Shinya H. Definitive treatment of malignant polyps of the colon. Ann Surg 1975; 182:516-24.

30. Colacchio TA, Forde KA, Scantlebury VP. Endoscopic polypectomy: inadequate treatment for invasive colorectal carcinoma. Ann Surg 1981; 194:704-07.

31. Nivatvongs S, Goldberg SM. Management of patients who have polyps containing invasive carcinoma removed via colonoscope. Dis Colon Rectum 1978; 21(1):8-11.

32. Teixeira CR, Mucenic M. Câncer Colorretal Precoce. Magalhães F, Cordeiro FT, Quilici F, Machado G, Amarante HM, 
Prolla JC, Leitão OR, Alves PRA, Sakai P. Endoscopia Digestiva Diagnóstica e Terapêutica (Livro da SOBED). $1^{\text {a }}$ ed. Rio de Janeiro: Revinter; 2004. 533-7.

33. Tanaka S, Haruma K, Oh-E H, Nagata S, Hirota Y, Furudoi A et al. Conditions of curability after endoscopic resection for colorectal carcinoma with submucosally massive invasion. Oncology Reports 2000; 7(4):783-8.

34. Moreira LF, Teixeira CR. Metástase linfonodal em tumores precoces do reto. Arq Gastroenterol 1992; 29:51-5.

35. Johnson DA, Guiney MS, Volpe RJ, Jones DM, Van Ness MM, Chobanian SJ et al. A prospective study of the prevalence of colonic neoplasms in asymptomatic patients with an age-related risk. Am J Gastroenterol 1990; 85:969-75.

36. Rex DK, Lehman GA, Hawes RH, Ulbright TM, Smith JJ. Screening colonoscopy in asymptomatic average-risk persons with negative fecal occult blood tests. Gastroentology 1991; 100:64-70.

37. Lieberman DA, Weiss DG, Bond JH, Ahnen DJ, Garewal H, Chejfec G. Use of colonoscopy to screen asymptomatic adults for colorectal cancer. N Engl J Med 2000; 343:162-8.

38. Demers RY, Neale AV, Budder H, Schade WJ. Pathologist agreement in the interpretation of colorectal polyps. AM J Gastroenterol 1990; 85:417-21.

39. Cross SS, Betmouni S, Burton JL, Dube AK, Feeley KM, Halbrook MR et al. What levels of agreement can be expected between histopathologists assining cases to discrete nominal categories? A study of the diagnosis of hyperplastic and adenomatous colorectal polyps. Mod Pathol 2000; 13(9):941-4.

40. Teixeira CR. Colonoscopia com Magnificação de Imagem e Cromoscopia das Lesões Colorretais. Magalhães F, Cordeiro FT, Quilici F, Machado G, Amarante HM, Prolla JC, Leitão OR, Alves PRA, Sakai P. Endoscopia Digestiva Diagnóstica e Terapêutica (Livro da SOBED). Rio de Janeiro: Revinter; 2004. 114-9.

41. Kudo S, Hirota S, Nakajima T, Hosobe S, Kusaka H, Kobayashi T et al. Colorectal tumours and pit pattern. Clin Pathol 1994; 47(10):880-5.

42. Kudo S, Tamura S, Nakajima T, Yamano H, Kusako H, Watanabe $\mathrm{H}$ et al. Diagnosis of colorectal tumorous lesions by magnifying endoscopy. Gastrointestinal Endosc 1996; 44(1):814.

43. Kudo S, Rubio CA, Teixeira CR, Kashida H, Kogure E. Pit pattern in colorectal neoplasia: endoscopic magnifying view. Endoscopy 2001; 33(4):367-73.
44. Konishi K, Kaneko K, Kurahashi T, Yamamoto T, Kushima M, kanda A et al. A Comparison of magnifying and nonmagnifying colonoscopy for diagnosis of colorectal polyps: A prospective study. Gastrointestinal Endoscopy 2003; 57(1):48-53.

45. Hurlstone DP, Cross SS, Adam I, Shorthouse AJ, Brown S, Sanders DS et al. Efficacy of high magnification chromoscopy colonoscopy for the diagnosis of neoplasia in flat and depressed lesions of the colorectum: a prospective analysis. Gut 2004; 53(2):384-290.

46. Konishi F, Morson BC. Pathology of colorectal adenomas: a colonoscopy survey. J Clin Pathol 1982; 35:830-41.

47. O'Brien MJ, Winawer SJ, Zauber AG, Gottlieb LS, Stemberg SS, Diaz B et al. National Polyp Study: patient and polyp characteristics associated with high grade dysplasia in colorectal adenomas. Gastroenterology 1990; 98(2):371-9.

48. Yoon H, Martin A, Benamouzig R, Longchampt E, Deyra Jacques, Chaussade $S$ et al. Inter-observer agreement on histological diagnosis of colon polyps: The APACC study 2002;26(3):220-4.

49. Fenger C, Bak M, Kronborg O, Svanholm H. Observer reproducibility in grading dysplasia in colorectal adenomas: comparison between two different grading systems. J Clin Pathol 1990; 43:320-4.

50. Troxel DB. Error in Surgical Pathology. Am J Surg Pathol 2004; 28:1092-5.

51. Ramsay AD. Errors in histopathology reporting: detection and avoidance. Histopathology 1999; 34:481-90.

52. Prescott RJ, Wells S, Bisset DL, Banerjee SS, Harris M. Audit of tumour histopathology reviewed by a regional oncology center. J Clin Pathol 1995; 48: 245-9.

53. Cramer SF. International Variation in Histologic Grading is Large and Persistent Feedback Does Not Improve Reproducibility. Am J Surg Pathol 2004; 28(2): 273-4.

\author{
Endereço para correspondência: \\ MARLISE MELLO CERATO \\ Rua Mariante, 239/conjunto 401 \\ Bairro: Moinhos de Vento \\ CEP: 90430-181 \\ Fone: (51) 3222-4789 \\ Fone/fax: (51) 3222-7797 \\ E-mail: mcerato@hotmail.com
}

